\title{
Relação entre adesão ao tratamento e qualidade de vida: revisão integrativa da literatura
}

\author{
Relationship between treatment adherence and quality of life: integrative literature review
}

Relación entre adhesión al tratamiento y calidad de vida: revisión integrativa de la literatura

Samilly Márjore Dantas Liberato ${ }^{1}$, Amanda Jéssica Gomes de Souza르. Andréa Tayse de Lima Gomes ${ }^{3}$, Lays Pinheiro de Medeiros ${ }^{4}$, Isabelle Katherinne Fernandes Costa ${ }^{5}$, Gilson de Vasconcelos Torres ${ }^{6}$

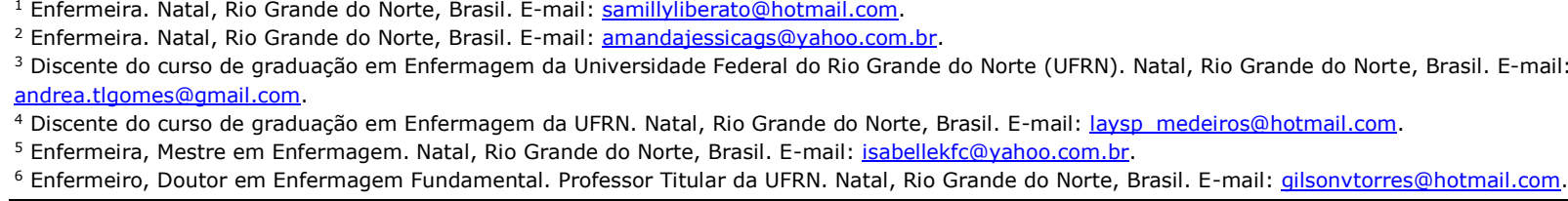

\section{RESUMO}

O presente estudo tem como objetivo investigar as relações entre adesão ao tratamento e qualidade de vida que têm sido publicadas na literatura científica. Trata-se de uma revisão integrativa realizada nas bases de dados Biblioteca Virtual em Saúde, SCOPUS, ISI Web of Knowledge e CINAHL, incluindo artigos publicados de 2007 a 2012 . A partir dos critérios de inclusão e exclusão, foram selecionados 14 artigos. Predominaram os artigos que estudaram adultos (61,5\%) e com nível de evidência VI (69,2\%). O número de participantes envolvidos nos estudos variou de 25 a 1.860 . Todos abordaram doenças crônicas. Do total de artigos, 69,2\% dos estudos encontraram relação significativa entre adesão ao tratamento e qualidade de vida. Os estudos apontaram que existe relação entre adesão ao tratamento e qualidade de vida, porém não foi possível definir a causalidade do fenômeno. Mesmo nos estudos em que não houve significância estatística, esta relação se mostrou positiva.

Descritores: Cooperação do Paciente; Qualidade de Vida; Assistência Integral à Saúde.

\section{ABSTRACT}

The objective of the present study is to investigate the relationships between treatment adherence and quality of life that have been published in scientific literature. This is an integrative review performed on the Virtual Health Library database, SCOPUS, ISI Web of Knowledge and CINAHL, including articles published in the period between 2007 and 2012. Considering the inclusion and exclusion criterion, 14 articles were selected. Most articles studied adults (61.5\%) with evidence level VI (69.2\%). The number of participants involved in the studies ranged between 25 and 1,860. All studies were on chronic diseases. Of all articles, $69.2 \%$ of the studies found a significant relationship between treatment adhesion and quality of life. The studies pointed at a relationship between treatment adherence and quality of life, but it was not possible to determine the causality of the phenomenon. Including the studies that found no statistical significance, the referred relationships proved positive.

Descriptors: Patient Compliance; Quality of Life; Comprehensive Health Care.

\section{RESUMEN}

Se objetiva investigar las relaciones entre adhesión al tratamiento y calidad de vida publicadas en la literatura científica. Revisión integrativa realizada en las bases de datos Biblioteca Virtual en Salud, SCOPUS, ISI Web of Knowledge y CINAHL, incluyendo artículos publicados de 2007 a 2012. De acuerdo a los criterios de inclusión y exclusión, fueron seleccionados 14 artículos. Predominaron los artículos que estudiaron a los adultos $(61,5 \%)$, con nivel de evidencia VI $(69,2 \%)$. El número de participantes involucrados en los estudios varió de 25 a 1860 . Todos abordaron enfermedades crónicas. Del total de artículos, 69,2\% encontró relación significativa entre adhesión al tratamiento y calidad de vida. Los estudios expresaron que existe relación significativa entre adhesión al tratamiento y calidad de vida, aunque no fue posible definir la causalidad del fenómeno. Incluso en los estudios en los que no hubo significatividad estadística, esta relación se mostró positiva.

Descriptores: Cooperación del Paciente; Calidad de Vida; Atención Integral de Salud. 


\section{INTRODUÇÃo}

A adesão terapêutica refere-se ao grau de concordância entre o comportamento de uma pessoa e as prescrições da equipe de saúde ${ }^{(1)}$. O conceito remete a ideia de uma relação de colaboração entre paciente e médico na tomada de decisões sobre o tratamento. Dessa forma, o termo adesão tem sido o eleito por diversos autores como o ideal para definir o fenômeno, por expressar participação voluntária e ativa por parte do usuário no desenvolvimento e ajuste do plano de cuidados $^{(2-5)}$.

Trata-se de um fenômeno complexo, pois o tratamento não se restringe à terapia medicamentosa e ao seguimento das prescrições dos profissionais de saúde. A Organização Mundial de Saúde (OMS) elencou cinco dimensões que interferem na adesão do paciente ao tratamento considerando a multidimensionalidade do processo: fatores relacionados ao paciente, ao tratamento, socioeconômicos, relacionados aos sistemas de saúde e a doença(1).

A baixa adesão ao tratamento pode ser traduzida em diversos comportamentos: dificuldades para seu início, suspensão prematura do tratamento, omissões ou esquecimentos de medicamentos, faltas às consultas ou retornos agendados, automedicação, ausência de modificação no estilo de vida ou hábitos necessários para a recuperação da enfermidade(6). A importância do problema de falta de adesão terapêutica é indiscutível se forem analisados os impactos que isso tem sob o ponto de vista clínico, médico, econômico e psicossocial( ${ }^{(7-8)}$.

Assim, torna-se um problema sério para a saúde pública contemporânea, especialmente quando se trata de doenças crônicas, uma vez que aumenta os custos com os cuidados com a saúde, pois diminui a eficácia e aumenta a possibilidade do fracasso do tratamento, eleva a possibilidade de complicações e diminui a qualidade de vida das populações ${ }^{(6-9)}$.

O conceito de qualidade de vida (QV) vem se destacando cada vez mais na sociedade atual, por sua relação direta com a saúde humana, tornando-se um tema de interesse coletivo. Contudo é uma temática complexa, subjetiva, que gera um leque ampliado de $\operatorname{significados}^{(10-}$ 11).

Segundo a OMS, entende-se por qualidade de vida "a percepção do indivíduo acerca de sua posição na vida, de acordo com o seu contexto cultural e sistema de valores nos quais vive"(12). Trata-se, portanto, de um conceito marcado pela subjetividade e multidimensionalidade, uma vez que envolve os componentes essenciais da condição humana, sejam eles físicos, psicológicos, sociais, culturais ou espirituais ${ }^{(13)}$.

A valorização do conceito de qualidade de vida reflete numa crescente preocupação com o paciente, o qual é visto holisticamente. O objetivo do tratamento deixa de focar exclusivamente a cura e passa a ser a reintegração do pacientes com o máximo de condições de ter uma vida normal, com qualidade e saúde ${ }^{(14)}$.

Assim, julga-se necessário a busca pelo aumento da compreensão do fenômeno da adesão, da qualidade de vida e a relação existente entre elas, com vistas a subsidiar e auxiliar uma mudança na prática de atenção à saúde superando a visão simplista e limitada da assistência.

Frente ao exposto, o presente estudo tem como objetivo investigar as relações entre adesão ao tratamento e qualidade de vida que têm sido publicadas na literatura científica.

\section{MÉTODOS}

Trata-se de uma revisão integrativa da literatura que se caracteriza como uma análise ampla da literatura sobre um assunto específico com o intuito de construir uma conclusão síntese de estudos realizados separadamente, mas que investigam problemas idênticos ou similares ${ }^{(15-}$ 16).

Para a realização desse estudo foram obedecidas as etapas expostas a seguir: elaboração da pergunta norteadora, estabelecimento dos objetivos da revisão e critérios de inclusão e exclusão dos artigos; definição das informações a serem extraídas das pesquisas; seleção dos artigos na literatura; análise dos resultados; discussão dos achados e apresentação da revisão ${ }^{(17)}$.

Como forma de conduzir esta revisão foi formulada a seguinte questão direcionadora: as relações entre adesão ao tratamento e qualidade de vida estudadas na literatura são significativas?

A busca pelas publicações foi realizada no mês de setembro de 2012 nas bases de dados Biblioteca Virtual em Saúde (BVS), SCOPUS, ISI Web of Knowledge e Cumulative Index to Nursing and Allied Health Literature (CINAHL).

Para o refinamento adequado dos artigos foi definida uma amostra, obedecendo aos seguintes critérios de inclusão: publicações em português, inglês e espanhol; disponíveis gratuitamente em texto completo nas bases de dados supracitadas; publicadas no período janeiro de 2007 a agosto de 2013, e artigos que abordassem relações entre adesão ao tratamento e qualidade de vida. Foram excluídos artigos publicados em outros idiomas, 
estudos em formato de editorial, carta ao editor ou revisão de literatura e artigos que não abordassem o tema proposto.

Para o levantamento das publicações na BVS foram utilizados os descritores controlados dos descritores em ciências da saúde (DeCS): "Adesão do paciente" e "Qualidade de vida". O cruzamento desses descritores na base citada ocorreu através do operador booleano AND. Nas bases SCOPUS, Isi Web of Knowledge e CINAHL foram escolhidos os descritores controlados do vocabulário $\mathrm{MeSH}$ - Medical Subject Headings, na língua inglesa: "Patient Adherence" e "Quality of Life". Nesse caso também foi empregado o operador booleano AND. Além disso, na SCOPUS utilizou-se a ferramenta "Article title, Abstract, Keywords".

Após o procedimento da busca eletrônica nas bases de dados mencionadas, as publicações foram pré- selecionadas com base na leitura do título e resumo. Após a leitura na íntegra dos artigos previamente selecionados, foram identificados os artigos que compuseram a amostra final desta revisão integrativa.

Com a busca computadorizada na base de dados BVS, foram encontrados 1.140 estudos e selecionados 10 artigos, sendo todos na MEDLINE. Na SCOPUS foram localizados 176 artigos, sendo que destes três fizeram parte da amostra final. Na ISI Web of Knowledge, 118 publicações foram apresentadas e uma foi selecionada para o estudo. A partir da pesquisa na base de dados CINAHL, 27 estudos foram localizados, porém nenhum foi incluído nesta revisão.

A Figura 1 apresenta o fluxograma referente ao processo de seleção dos artigos que fizeram parte da amostra final desta revisão.

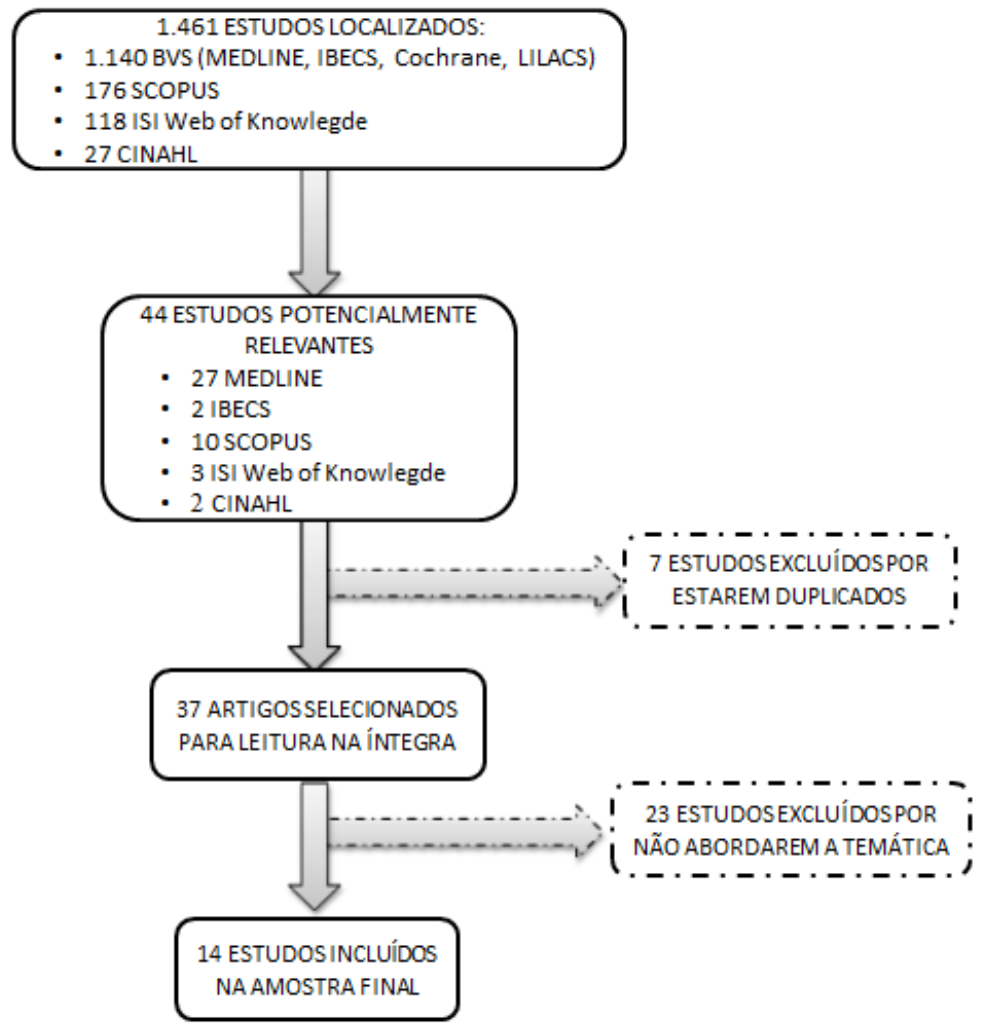

Figura 1. Fluxograma representativo do processo de seleção dos artigos.

Para a coleta de dados dos artigos, utilizou-se um instrumento validado, adaptado para este estudo, que engloba a identificação do artigo e características

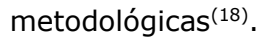

Os artigos selecionados foram classificados em relação ao nível de evidência, sendo que para tal é considerado o delineamento de pesquisa utilizado para o desenvolvimento do estudo. Nesta revisão foi empregado um sistema de classificação composto por sete níveis, sendo: Nível I - evidências oriundas de revisões sistemáticas ou meta-análise de relevantes ensaios clínicos; Nível II - evidências derivadas de pelo menos um ensaio clínico randomizado controlado bem delineado; Nível III - ensaios clínicos bem delineados sem randomização; Nível IV - estudos de coorte e de casocontrole bem delineados; Nível V - revisão sistemática de estudos descritivos e qualitativos; Nível VI - evidências derivadas de um único estudo descritivo ou qualitativo e 
Nível VII - opinião de autoridades ou relatório de comitês de especialistas ${ }^{(19)}$. As evidências pertencentes aos níveis I e II são consideradas fortes, de III a $V$ evidências moderadas e VI e VII evidências fracas.

Após a leitura dos artigos, os dados foram digitados em planilhas eletrônicas, analisados por meio da estatística descritiva e apresentados sob a forma de quadros.

\section{RESULTADOS}

O Quadro 1 sintetiza as informações disponibilizadas pelos artigos que foram incluídos na revisão de literatura.

Quadro 1. Distribuição dos artigos segundo autor/base de dados, população/número de participantes, método de avaliação da adesão, instrumento de avaliação da QV, relação significativa da adesão com a QV, tipo de estudo/nível de evidência.

\begin{tabular}{|c|c|c|c|c|c|}
\hline $\begin{array}{c}\text { Autor/Base de } \\
\text { dados }\end{array}$ & $\begin{array}{l}\text { População } \\
\text { (Número de } \\
\text { Participantes) }\end{array}$ & $\begin{array}{l}\text { Método de } \\
\text { avaliação da } \\
\text { adesão }\end{array}$ & $\begin{array}{l}\text { Instrumento de } \\
\text { avaliação da QV }\end{array}$ & $\begin{array}{c}\text { Relação } \\
\text { significativa da } \\
\text { adesão com a QV }\end{array}$ & $\begin{array}{c}\text { Tipo de Estudo / } \\
\text { Nível de } \\
\text { evidência }\end{array}$ \\
\hline $\begin{array}{c}\text { Puschner B, } \\
\text { Angermeyer MC, } \\
\text { Leese M, } \\
\text { Thornicroft G, } \\
\text { Schene A, Kikkert } \\
\text { M, et al (20) / ISI } \\
\text { Web of Knowledge }\end{array}$ & $\begin{array}{c}\text { Adultos com } \\
\text { esquizofrenia } \\
\text { (373) }\end{array}$ & $\begin{array}{c}\text { Medication } \\
\text { Adherence } \\
\text { Questionnaire } \\
\text { (MAQ) }\end{array}$ & $\begin{array}{l}\text { Manchester Short } \\
\text { Assessment of } \\
\text { Quality of Life }\end{array}$ & SIM & $\begin{array}{c}\text { Ensaio Clínico } \\
\text { Randomizado / II }\end{array}$ \\
\hline $\begin{array}{c}\text { Margalho R, } \\
\text { Pereira M, } \\
\text { Ouakinin S, } \\
\text { Canavarro MC }{ }^{(21)} / \\
\text { SCOPUS }\end{array}$ & $\begin{array}{l}\text { Adultos infectados } \\
\text { pelo HIV (762) }\end{array}$ & $\begin{array}{l}\text { Ficha de dados } \\
\text { clínicos }\end{array}$ & WHOQOL-HIV-Bref & SIM & Analítico / VI \\
\hline $\begin{array}{c}\text { Marcellin } \mathrm{P}, \\
\text { Chousterman M, } \\
\text { Fontanges T, } \\
\text { Ouzan D, Rotily M, } \\
\text { Varastet M, et al } \\
(22) / \text { SCOPUS }\end{array}$ & $\begin{array}{c}\text { Adultos com } \\
\text { Hepatite } C(1860)\end{array}$ & $\begin{array}{l}\text { Auto-relato do } \\
\text { paciente }\end{array}$ & $\begin{array}{l}\text { Short Form Health } \\
\text { Survey } 36 \text { (SF-36) }\end{array}$ & SIM & $\begin{array}{c}\text { Estudo de Coorte / } \\
\text { IV }\end{array}$ \\
\hline $\begin{array}{l}\text { Adelufosi AO, } \\
\text { Adebowale TO, } \\
\text { Abayomi O, } \\
\text { Mosanya JT(23) / } \\
\text { MEDLINE }\end{array}$ & $\begin{array}{c}\text { Adultos com } \\
\text { esquizofrenia } \\
\text { (313) }\end{array}$ & $\begin{array}{c}\text { Medication } \\
\text { Adherence } \\
\text { Questionnaire } \\
\text { (MAQ) }\end{array}$ & WHOQOL-BREF & SIM & Analítico / VI \\
\hline $\begin{array}{c}\text { Akman B, Uyar M, } \\
\text { Afsar B, Sezer S, } \\
\text { Ozdemir FN, } \\
\text { Haberal M (24) / } \\
\text { MEDLINE }\end{array}$ & $\begin{array}{l}\text { Adultos na lista de } \\
\text { espera para } \\
\text { transplante renal } \\
\text { (86) }\end{array}$ & $\begin{array}{c}\text { Parâmetros } \\
\text { estabelecidos pelos } \\
\text { pesquisadores }\end{array}$ & $\begin{array}{l}\text { Short Form Health } \\
\text { Survey } 36 \text { (SF-36) }\end{array}$ & SIM & Analítico / VI \\
\hline \multirow{3}{*}{$\begin{array}{l}\text { Cotugno G, Nicolò } \\
\text { R, Cappelletti S, } \\
\text { Goffredo BM, } \\
\text { Dionisi Vici C, V Di } \\
\text { Ciommo V (25) / } \\
\text { MEDLINE }\end{array}$} & \multirow{3}{*}{$\begin{array}{c}\text { Crianças e } \\
\text { adolescentes com } \\
\text { fenilcetonúria ( } 41 \text { ) }\end{array}$} & \multirow{3}{*}{$\begin{array}{l}\text { Níveis séricos de } \\
\text { fenilalanina }\end{array}$} & $\begin{array}{c}\text { Child Health } \\
\text { Questionnaire } \\
(\mathrm{CHQ})\end{array}$ & \multirow{3}{*}{ NÃO } & \multirow{3}{*}{ Analítico / VI } \\
\hline & & & $\begin{array}{c}\text { CHQ-PF (versão } \\
\text { para os pais) }\end{array}$ & & \\
\hline & & & $\begin{array}{l}\text { Short Form Health } \\
\text { Survey } 36 \text { (SF-36) }\end{array}$ & & \\
\hline $\begin{array}{c}\text { Fisak B, Belkin MH, } \\
\text { von Lehe AC, } \\
\text { Bansal MM (26) / } \\
\text { MEDLINE }\end{array}$ & $\begin{array}{c}\text { Crianças e } \\
\text { adolescentes com } \\
\text { anemia falciforme } \\
(78)\end{array}$ & $\begin{array}{l}\text { Adherence \& Self- } \\
\text { Care Inventory }\end{array}$ & $\begin{array}{l}\text { Pediatric Quality of } \\
\text { Life Inventory }\end{array}$ & SIM & Analítico / VI \\
\hline \multirow{4}{*}{$\begin{array}{c}\text { Fredericks EM, } \\
\text { Magee JC, Opipari- } \\
\text { Arrigan L, Victoria } \\
\text { Shieck V, Well A, } \\
\text { Lopez MJ (27)/ } \\
\text { MEDLINE }\end{array}$} & \multirow{4}{*}{$\begin{array}{l}\text { Adolescentes que } \\
\text { receberam } \\
\text { transplante de } \\
\text { fígado (25) }\end{array}$} & $\begin{array}{c}\text { Medication } \\
\text { Adherence } \\
\text { Measure (MAM) } \\
\end{array}$ & $\begin{array}{l}\text { Pediatric Quality of } \\
\text { Life Inventory }\end{array}$ & \multirow{4}{*}{$\begin{array}{l}\text { A relação foi } \\
\text { significativa } \\
\text { apenas quando a } \\
\text { adesão foi avaliada } \\
\text { pelos níveis séricos } \\
\text { da medicação }\end{array}$} & \multirow{4}{*}{ Analítico / VI } \\
\hline & & $\begin{array}{l}\text { Níveis séricos da } \\
\text { medicação }\end{array}$ & $\begin{array}{l}\text { Child Health } \\
\text { Questionnaire } \\
(\mathrm{CHQ})\end{array}$ & & \\
\hline & & $\begin{array}{l}\text { Entrevista clínica } \\
\text { conduzida }\end{array}$ & \multirow{2}{*}{$\begin{array}{c}\text { CHQ-PF (versão } \\
\text { para os pais) }\end{array}$} & & \\
\hline & & $\begin{array}{c}\text { Taxa de } \\
\text { atendimento }\end{array}$ & & & \\
\hline $\begin{array}{c}\text { Gorevski E, Succop } \\
\text { P, Sachdeva J, } \\
\text { Cavanaugh TM, } \\
\text { Volek P, Heaton P } \\
\text { et al }{ }^{(28)} / \text { SCOPUS }\end{array}$ & $\begin{array}{c}\text { Adultos } \\
\text { submetidos a } \\
\text { transplante de rim } \\
\text { (86) e de fígado } \\
\text { (50) }\end{array}$ & $\begin{array}{c}\text { Immunosuppressiv } \\
\text { e Therapy } \\
\text { Adherence Scale. }\end{array}$ & $\begin{array}{l}\text { Short Form Health } \\
\text { Survey } 36 \text { (SF-36) }\end{array}$ & NÃO & Analítico / VI \\
\hline
\end{tabular}




\begin{tabular}{|c|c|c|c|c|c|}
\hline $\begin{array}{c}\text { Autor/Base de } \\
\text { dados }\end{array}$ & $\begin{array}{c}\text { População } \\
\text { (Número de } \\
\text { Participantes) }\end{array}$ & $\begin{array}{c}\text { Método de } \\
\text { avaliação da } \\
\text { adesão }\end{array}$ & $\begin{array}{l}\text { Instrumento de } \\
\text { avaliação da QV }\end{array}$ & $\begin{array}{c}\text { Relação } \\
\text { significativa da } \\
\text { adesão com a QV }\end{array}$ & $\begin{array}{c}\text { Tipo de Estudo / } \\
\text { Nível de } \\
\text { evidência }\end{array}$ \\
\hline $\begin{array}{c}\text { Holmes WC, Bilker } \\
\text { WB, Wang H, } \\
\text { Chapman J, Gross } \\
\text { R (29) / MEDLINE }\end{array}$ & $\begin{array}{l}\text { Adultos infectados } \\
\text { pelo HIV (116) }\end{array}$ & $\begin{array}{c}\text { Medication Event } \\
\text { Monitoring System } \\
\text { [MEMS] }\end{array}$ & $\begin{array}{c}\text { HIV/AIDS- } \\
\text { Targeted Quality of } \\
\text { Life (HAT-QoL) }\end{array}$ & NÃO & $\begin{array}{c}\text { Estudo de Coorte / } \\
\text { VI }\end{array}$ \\
\hline \multirow{2}{*}{$\begin{array}{c}\text { Martínez YV, } \\
\text { Prado-Aguilar CA, } \\
\text { Rascón-Pacheco } \\
\text { RA, Valdivia- } \\
\text { Martínez JJ (30) / } \\
\text { MEDLINE }\end{array}$} & \multirow{2}{*}{$\begin{array}{c}\text { Adultos com } \\
\text { diabetes tipo } 2 \\
(238)\end{array}$} & $\begin{array}{l}\text { Attitude to } \\
\text { Treatment } \\
\text { Adherence }\end{array}$ & \multirow{2}{*}{ WHOQOL-100 } & \multirow{2}{*}{ NÃO } & \multirow{2}{*}{ Analítico / VI } \\
\hline & & $\begin{array}{l}\text { Contagem de } \\
\text { comprimidos }\end{array}$ & & & \\
\hline \multirow{3}{*}{$\begin{array}{c}\text { Nachman F, } \\
\text { Campo MP, } \\
\text { González A, Corzo } \\
\text { L, Vázquez H, } \\
\text { Sfoggia C, et al (31) } \\
\text { / MEDLINE }\end{array}$} & \multirow{3}{*}{$\begin{array}{c}\text { Adultos com } \\
\text { doença celíaca } \\
\text { (53) }\end{array}$} & $\begin{array}{l}\text { Auto-relato do } \\
\text { paciente }\end{array}$ & \multirow{3}{*}{$\begin{array}{l}\text { Short Form Health } \\
\text { Survey } 36 \text { (SF-36) }\end{array}$} & \multirow{3}{*}{ SIM } & \multirow{3}{*}{ Caso-Controle / IV } \\
\hline & & Opinião do Médico & & & \\
\hline & & $\begin{array}{l}\text { Avaliação com } \\
\text { nutricionista }\end{array}$ & & & \\
\hline $\begin{array}{c}\text { Rivett L, Rothberg } \\
\text { A, Stewart A, } \\
\text { Berkowitz R }{ }^{(32)} / \\
\text { MEDLINE }\end{array}$ & $\begin{array}{c}\text { Adolescentes com } \\
\text { escoliose idiopática } \\
\text { (31) }\end{array}$ & $\begin{array}{c}\text { Parâmetros } \\
\text { estabelecidos pelos } \\
\text { pesquisadores }\end{array}$ & $\begin{array}{c}\text { The Brace } \\
\text { Questionnaire } \\
\text { (BrQ) }\end{array}$ & SIM & Analítico / VI \\
\hline $\begin{array}{c}\text { Wagner G, Berger } \\
\text { G, Sinnreich U, } \\
\text { Grylli V, Schober } \\
\text { E, Huber WD, et al } \\
\text { (33) / MEDLINE }\end{array}$ & $\begin{array}{c}\text { Adolescentes com } \\
\text { doença celíaca } \\
\text { (283) }\end{array}$ & $\begin{array}{l}\text { Auto-relato do } \\
\text { paciente }\end{array}$ & $\begin{array}{l}\text { Inventory of Life } \\
\text { Quality in Children } \\
\text { and Adolescents } \\
\text { (ILC) }\end{array}$ & NÃO & Caso-Controle / IV \\
\hline
\end{tabular}

Dos 14 artigos inclusos na revisão, nove analisaram adultos, três tiveram foco em adolescentes e dois investigaram crianças e adolescentes. O número de participantes envolvidos nos estudos variou de 25 a 1.860. Todos abordaram doenças crônicas.

De acordo com o Quadro 1, predominaram os estudos com nível VI de evidência científica (64,3\%). Pode-se perceber também que, tanto os métodos que investigaram a adesão quanto os instrumentos que avaliaram a QV, foram amplamente diversificados. O Quadro 1 ilustra ainda que $64,3 \%$ dos estudos encontraram relação significativa entre adesão ao tratamento e qualidade de vida.

\section{DISCUSSÃO}

Entre um total de 14 artigos analisados, cinco não encontraram relação significativa entre adesão ao tratamento e qualidade de vida ${ }^{(25,28-30,33)}$. No entanto, um dos referidos estudos identificou que a combinação de conhecimento adequado e atitude positiva frente ao tratamento apresentou relação significativa com todos os domínios da QV avaliados ${ }^{(30)}$. Com base nesses resultados evidencia-se a importância de estimular o conhecimento do paciente sobre seu tratamento, incentivar o mesmo a expressar suas percepções e explorar fatores psicológicos envolvidos.

Um conhecimento adequado sobre o tratamento medicamentoso exige a definição do nome do medicamento, a finalidade, o esquema de administração, efeitos adversos ou colaterais e instruções para situações especiais(34). Essa constatação remete à valorização do profissional enfermeiro uma vez que este desenvolve uma prática singular de educação em saúde visando a promoção da adesão ao tratamento e a redução dos riscos associados ao uso inadequado dos medicamentos ${ }^{(35)}$.

Estudo realizado com 762 adultos infectados pelo HIV revelou que o grupo de pessoas classificadas como não aderentes apresentaram piores resultados de QV, sendo esta relação significativa em quatro dos seis domínios avaliados pelo WHOQOL-HIV-Bref ${ }^{(21)}$. Em contraste com esses resultados, outro estudo incluído nesta revisão que investigou 116 pessoas infectadas pelo HIV através do HIV/AIDS-Targeted Quality of Life (HAT-QoL), não identificou relação estatística significativa entre adesão e $\mathrm{QV}^{(29)}$.

O desenvolvimento da terapia antirretroviral configurou-se como fato marcante na história desse agravo, pois embora não represente a cura, permitiu uma diminuição considerável nas manifestações clínicas associadas ao HIV. Ao retardar o surgimento do quadro de imunodeficiência e melhorar a capacidade imunológica da pessoa infectada, a terapia promove a qualidade de vida ao mesmo tempo em que proporciona longevidade aos portadores ${ }^{(36)}$

No cenário da Nigéria, pacientes com esquizofrenia foram investigados, sendo classificados como aderentes 
$(59,7 \%)$ e não aderentes $(40,3 \%)$ de acordo com o teste de Morisky. Quando relacionados com a QV, os escores médios do grupo aderente foram significativamente maiores do que os escores médios do grupo não aderente em todos os domínios avaliados pelo WHOQOL-BREF ( $\mathrm{p} \leq$ $0,001)^{(23)}$.

Corroborando com esses dados, outro estudo desenvolvido com pacientes com esquizofrenia, realizado em quatro países da Europa, também encontrou relação significativa entre adesão ao tratamento e QV. Além disso, essa investigação ainda observou essa mesma relação na avaliação realizada após um ano do início do estudo(20).

Em um dos estudos os pacientes apresentaram uma piora temporária da QV durante o tratamento, porém seis meses após o término do tratamento a QV ficou em níveis um pouco maiores do que no início do tratamento(22). Nesse momento, é importante que o profissional tranquilize o paciente, esclareça dúvidas, incentivando-o a manter a terapia.

Frente ao exposto, há de se considerar a importância da relação profissional de saúde-paciente ser pautada em ampla comunicação. Uma pesquisa envolvendo 26 pacientes com úlceras venosas demonstrou que os pacientes que mantiveram uma relação de confiança com o seu enfermeiro apresentaram melhor adesão às modificações no estilo de vida(37).

Deve-se considerar ainda que a adesão pode estar relacionada também à qualidade dos serviços nos quais os pacientes estão sendo acompanhados ${ }^{(38)}$. De acordo com uma investigação, a adesão se mostrou menor entre os grupos de pacientes acompanhados em serviços com piores níveis de qualidade ${ }^{(39)}$.

A temática transplante foi o contexto de três estudos: um deles investigou pacientes que estavam na fila de espera para transplante renal, outro, pacientes submetidos a transplante renal e de fígado e o terceiro, pacientes que já haviam recebido transplante de fígado. No primeiro estudo, o grupo "não-adesão" apresentou escores de QV com valor médio de 80.8. Já no grupo "adesão" esse valor foi de 92.2. Ao aplicar os testes estatísticos a diferença entre os grupos foi significante ( $p$ $<0,04)^{(24)}$.

A investigação realizada com pacientes submetidos a transplante renal e de fígado não identificou relação estatisticamente significante entre adesão à terapia imunossupressora e qualidade de vida, todavia os autores apontaram como possível razão o tamanho pequeno da amostra ${ }^{(28)}$. No caso dos pacientes que já haviam recebido transplante de fígado foi aplicada uma análise multimétodo da adesão (MAM, níveis séricos da medicação, entrevista clínica conduzida e taxa de atendimento). Com a aplicação de testes estatísticos, apenas o nível sérico da medicação teve relação significativa com a $\mathrm{QV}^{(27)}$, porém os próprios autores elucidaram a limitação do estudo pela falta de consenso entre qual a medida de adesão mais adequada.

Diversos métodos podem ser utilizados para verificar adesão ao tratamento. Entretanto, devido a complexidade do conceito de adesão, são raros os instrumentos que se propõem e são realmente capazes de avaliar o fenômeno em sua totalidade. A combinação de métodos que se correlacionem com a multidimensionalidade do processo de adesão é a proposta que resultará na melhor aproximação para medir este comportamento(1).

Frequentemente, a literatura apresenta estudos que evidenciam uma preocupação com a adesão aos medicamentos em detrimento da adesão terapêutica. Tendo em vista que o gerenciamento das condições crônicas demanda uma adaptação contínua e mudanças no estilo de vida, a falta de controle de uma doença não pode ser atribuída exclusivamente a não adesão à terapia medicamentosa ${ }^{(1,40)}$.

A doença celíaca foi o alvo de dois estudos: um envolvendo adultos $^{(31)}$ e outro com adolescentes ${ }^{(33)}$. Em comparação com os indivíduos parcialmente aderentes, os adultos classificados como aderentes tiveram escores significativamente melhores de qualidade de vida para seis das oito dimensões avaliadas pelo SF-36(31). No caso dos adolescentes, apesar do grupo aderente apresentar escores de QV maiores do que o grupo não-aderente, esta diferença não foi significativa( ${ }^{(33)}$.

Entre os cinco estudos que tiveram como população crianças e/ou adolescentes, três identificaram a relação entre adesão e QV como significativa e dois como não significativa, o que releva que o fenômeno da adesão nessa faixa etária não permite resultados conclusivos ${ }^{(25-}$ 27,32-33).

A dependência de cuidadores, que podem ou não estar doentes e ser ou não pais da criança; excessivo número de doses e drogas; pobre palatabilidade das drogas; dificuldade de tomar comprimidos e interferência com rotinas diárias, são fatores que configuram a adesão de crianças mais complexa quando comparada com pacientes adultos ${ }^{(41-42)}$.

Foi consenso entre os autores que são necessários mais estudos com essas populações que explorem melhor esta relação e investiguem os fatores que impedem a adesão, uma vez que a infância e adolescência são etapas do desenvolvimento humano repletas de peculiares. 


\section{CONSIDERAÇÕES FINAIS}

Fundamentados na análise desenvolvida, os estudos apontaram que existe relação entre adesão ao tratamento e qualidade de vida. Esta relação foi significativa na maioria dos estudos e mesmo naqueles em que não houve significância estatística esta relação se mostrou positiva.

Tal fato evidencia que a adesão ao tratamento deve ser mais valorizada pelos profissionais de saúde que almejam prestar aos usuários uma assistência pautada na visão holística do ser humano visando atingir o conceito ampliado de saúde.

Vale ressaltar que um elevado número de publicações foram excluídas da presente revisão, pois, apesar de avaliarem a adesão ao tratamento e a qualidade de vida, não exploraram a relação entre essas variáveis. Este cenário demonstra que o fenômeno está sendo

\section{REFERÊNCIAS}

1. Organização Mundial de Saúde. Cuidados inovadores para condições crônicas: componentes estruturais de ação: relatório mundial. Brasília (DF): 2003 [cited 2012 Oct 13]. Available from: http://www.who.int/diabetesactiononline/about/icccportuguese.p df.

2. Gomes-Vilas Boas LC. Apoio social, adesão ao tratamento e controle metabólico de pessoas com diabetes mellitus tipo 2.[Dissertação].São Paulo: Escola de Enfermagem /USP; 2009.

$171 \mathrm{p}$.

3. Chatterjee JS. From compliance to concordance in diabetes. J Med Ethics [Internet]. 2006 [cited 2012 set 16];32(9):507-10. Available from:

http://jme.bmj.com/content/32/9/507.full.pdf+html.

4. Gusmão JL, Mion Júnior D. Adesão ao tratamento - conceitos. Rev. bras. hipertens. [Internet]. 2006 [cited 2012 set $16] ; 13(1): 23-5$. Available from:

http://www.deciomion.com.br/medicos/Artigos/artigos_decio/Ad esao_ao tratamento_www-deciomion-com-br.pdf.

5. Osterberg L, Blaschke T. Adherence to medication. N Engl J Med. [Internet]. 2005 [cited 2012 set 16];353(5):487-97. Available from:

http://www.nejm.org/doi/pdf/10.1056/NEJMra050100. 6. Telles-Correia D, Barbosa A, Mega I, Monteiro E. Validação do questionário multidimensional da adesão no doente com transplante hepático. Acta med. port. [Internet]. 2008 [cited 2012 set 16$] ; 21(1): 31-6$. Available from: http://www.actamedicaportuguesa.com/pdf/2008-21/1/031036.pdf.

7. Camargo-Borges C, Japur M. Sobre a (não) adesão ao tratamento: ampliando sentidos do autocuidado. Texto \& contexto enferm. [Internet]. 2008 [cited 2012 set 16];17(1):6471. Available from: http://www.scielo.br/pdf/tce/v17n1/07.pdf. 8. Alfonso L M. Repercusiones para la salud pública de la adherencia terapéutica deficiente. Rev. cuba. salud pública. [Internet]. 2006 [cited 2012 set 16];32(3). Available from: http://bvs.sld.cu/revistas/spu/vol32 3 06/spu13306.htm. 9. Almeida HO, Versiani ER, Dias AR, Novaes MRCG, Trindade EMV. Adesão a tratamentos entre idosos. Com. Ciências Saúde [Internet]. 2007 [cited 2012 set 16];18(1):57-67. Available from: http://www.fepecs.edu.br/revista/Vol18 1art07.pdf. 10. Vido MB, Fernandes RAQ. Qualidade de Vida: considerações sobre conceito e instrumentos de medida. Online braz. j. nurs. [Intenet]. 2007 [cited 2012 set 16];6(2). Available from: http://www.objnursing.uff.br/index.php/nursing/login? source $=\%$ 2F\%2Findex.php\%2Fnursing\%2Farticle\%2Fview\%2Fj.16764285.2007.870\%2F197. subestimado, o que acaba se refletindo em conhecimentos inconclusivos, incapazes de fornecer melhores subsídios à relação.

Percebeu-se também que muitos autores sinalizaram a necessidade de mais estudos longitudinais serem realizados com o intuito de explorar a causalidade do fenômeno e aprofundar os conhecimentos sobre o impacto das consequências dessa relação ao longo do tempo.

Apesar de não fornecer evidências científicas consideradas fortes, o presente estudo permitiu destacar a importância do acompanhamento constante da adesão no tratamento. Analisar o fenômeno da adesão demonstra ser fundamental, tanto para a melhoria das políticas e práticas de saúde voltadas ao aprimoramento da efetividade da assistência prestada, quanto para a qualidade de vida do usuário.

11. Oliveira CJ, Pereira CAR, Pontes JNC, Fialho AVM, Moreira TMM. Análise da produção científica na área da saúde sobre qualidade de vida no Brasil entre 2000 e 2005: um estudo bibliográfico. Rev. Eletr. Enf. [Internet]. 2007 [cited 2012 set 16];9(2):496-05. Available from:

http://www.fen.ufg.br/revista/v9/n2/v9n2a16.htm

12. Organização Mundial de Saúde. Divisão de Saúde Mental Grupo WHOQOL. Versão em português dos instrumentos de avaliação de qualidade de vida (WHOQOL). Genebra: OMS; 1998 [cited 2012 oct 13]. Available from:

http://www.ufrgs.br/psiq/whogol.html

13. Ribeiro JP, Rocha AS, Popim RC. Compreendendo o significado de qualidade de vida segundo idosos portadores de diabetes mellitus tipo II. Esc. Anna Nery Rev. Enferm. [Internet]. 2010 [cited 2012 oct 13];14(4):765-71. Available from: http://www.scielo.br/pdf/ean/v14n4/v14n4a16.pdf 14. Santos RFFN, Porfírio GJM, Pitta GBB. A diferença na qualidade de vida de pacientes com doença venosa crônica leve e grave. ] Vasc Bras. [Internet]. 2009 [cited 2012 oct 13];8(2):143-7. Available from:

http://www.scielo.br/pdf/jvb/v8n2/a08v8n2.pdf 15. Barbosa LR, Melo MRAC. Relações entre qualidade da assistência de enfermagem: revisão integrativa da literatura. Rev. bras. enferm. [Internet]. 2008 [cited $2012 \mathrm{apr}$ 27];61(3):366-70. Available from:

http://www.scielo.br/pdf/reben/v61n3/a15v61n3.pdf 16. Mendes KDS, Silveira, RCCP, Galvão, CM. Revisão integrativa: método de pesquisa para a incorporação de evidências na saúde e na enfermagem. Texto \& contexto enferm. [Internet]. 2008 [cited 2012 apr 27];17(4):758-64. Available from: http://www.scielo.br/pdf/tce/v17n4/18.pdf

17. Souza MT, Silva MD, Carvalho R. Revisão integrativa: o que é e como fazer. Einstein [Internet]. 2010 [cited 2012 apr 27];8(1 Pt 1):102-6. Available from:

http://apps.einstein.br/revista/arquivos/PDF/1134Einsteinv8n1_p102-106_port.pdf

18. Ursi ES, Gavao CM. Prevenção de lesões de pele no perioperatório: revisão integrativa da literatura. Rev. latinoam. enferm. [Internet]. 2006 [cited 2012 may 04];14(1):124-131. Available from:

http://www.scielo.br/pdf/rlae/v14n1/v14n1a17.pdf 19. Stillwell S, Melnyk BM, Fineout-Overholt E, Williamson K. Evidence-based practice: step by step. Am J Nurs [Internet]. 2010[cited 2012 set 27];110(5):41-7. Available from: http://journals.Iww.com/ajnonline/Fulltext/2010/01000/Evidence Based Practice Step by Step The Seven.30.aspx 
20. Puschner B, Angermeyer MC, Leese M, Thornicroft G, Schene $A$, Kikkert $M$, et al. Course of adherence to medication and quality of life in people with schizophrenia. Psychiatry Res [Internet]. 2009 [cited 2012 set 16];165(3):224-33. Available from:

http://www.sciencedirect.com/science/article/pii/S01651781070 03654\#

21. Margalho R, Pereira M, Ouakinin S, Canavarro MC. Adesão à haart, qualidade de vida e sintomatologia psicopatológica em doentes infectados pelo VIH/SIDA. Acta Med Port. [Internet]. 2011 [cited 2012 set 16];24 Suppl 2:539-48. Available from: http://www.actamedicaportuguesa.com/pdf/201124/suplemento-originais/539-548.pdf

22. Marcellin $P$, Chousterman $M$, Fontanges $T$, Ouzan D, Rotily $M$, Varastet $M$, et al. Adherence to treatment and quality of life during hepatitis $C$ therapy: a prospective,real-life,observational study. Liver Int. [Internet]. 2011 [cited 2012 set 16];31(4):51624. Available from:

http://onlinelibrary.wiley.com/doi/10.1111/j.14783231.2011.02461.x/full

23. Adelufosi AO, Adebowale TO, Abayomi O, Mosanya JT. Medication adherence and quality of life among Nigerian outpatients with schizophrenia. Gen Hosp Psychiatry. [Internet]. 2011 [cited 2012 set 16];34(1):72-9. Available from: http://www.ghpjournal.com/article/S0163-8343(11)002908/fulltext.

24. Akman B, Uyar M, Afsar B, Sezer S, Ozdemir FN, Haberal M. Adherence, depression and quality of life in patients on a renal transplantation waiting list. Transpl Int. [Internet]. 2007 [cited 2012 set 16$] ; 20(8): 682-7$. Available from:

http://onlinelibrary.wiley.com/doi/10.1111/j.14322277.2007.00495.x/full.

25. Cotugno G, Nicolò R, Cappelletti S, Goffredo BM, Dionisi Vici C, V Di Ciommo V. Adherence to diet and quality of life in patients with phenylketonuria. Acta Paediatr. [Internet]. 2011 [cited 2012 set 16];100(8):1144-9. Available from: http://onlinelibrary.wiley.com/doi/10.1111/j.16512227.2011.02227.x/full.

26. Fisak B, Belkin MH, von Lehe AC, Bansal MM. The relation between health-related quality of life, treatment adherence and disease severity in a paediatric sickle cell disease sample. Child Care Health Dev. [Internet]. 2011 [cited 2012 set

16];38(2):204-10. Available from:

http://onlinelibrary.wiley.com/doi/10.1111/j.1365-

2214.2011.01223.x/full.

27. Fredericks EM, Magee JC, Opipari-Arrigan L, Victoria Shieck V, Well A, Lopez MJ. Adherence and health-related quality of life in adolescent liver transplant recipients. Pediatr Transplant. [Internet]. 2008 [cited 2012 set 16];12(3):289-99. Available from: http://onlinelibrary.wiley.com/doi/10.1111/j.13652214.2011.01223.x/full.

28. Gorevski E, Succop P, Sachdeva J, Cavanaugh TM, Volek P, Heaton $\mathrm{P}$, et al. Is there an association between immunosuppressant therapy medication adherence and depression, quality of life, and personality traits in the kidney and liver transplant population? Patient Prefer Adherence [Internet]. 2013 [cited 2013 set 25];16(7):301-7. Available from: http://www.ncbi.nlm.nih.gov/pmc/articles/PMC3630988/ 29. Holmes WC, Bilker WB, Wang H, Chapman J, Gross R. HIV/AIDS-Specific Quality of Life and Adherence to Antiretroviral Therapy Over Time. J Acquir Immune Defic Syndr [Internet]. 2007 [cited 2012 set 16];46(3):323-7. Available from: http://journals.Iww.com/jaids/Fulltext/2007/11010/HIV AIDS S pecific Quality of Life and Adherence to.11.aspx

30. Martínez YV, Prado-Aguilar CA, Rascón-Pacheco RA, ValdiviaMartínez JJ. Quality of life associated with treatment adherence in patients with type 2 diabetes: a cross-sectional study. BMC Health Serv Res. [Internet]. 2008 [cited 2012 set 16]; 8(7). Available from: http://www.biomedcentral.com/1472$6963 / 8 / 164$

31. Nachman F, Campo MP, González A, Corzo L, Vázquez H, Sfoggia $C$, et al. Long-term deterioration of quality of life in adult patients with celiac disease is associated with treatment noncompliance. Dig Liver Dis [Internet]. 2010 [cited 2012 set
16];42(10):685-91. Available from:

http://www.sciencedirect.com/science/article/pii/S15908658100 00952

32. Rivett L, Rothberg A, Stewart A, Berkowitz R. The relationship between quality of life and compliance to a brace protocol in adolescents with idiopathic scoliosis: a comparative study. BMC Musculoskelet Disord. [Internet]. 2009 [cited 2012 set 16$] ; 10(1): 1-6$. Available from: http://www.biomedcentral.com/1471-2474/10/5.

33. Wagner G, Berger G, Sinnreich U, Grylli V, Schober E, Huber WD, et al. Quality of Life in Adolescents With Treated Coeliac Disease: Influence of Compliance and Age at Diagnosis. J Pediatr Gastroenterol Nutr. [Internet]. 2008 [cited 2012 set

16];47(5):555-61. Available from:

http://journals.Iww.com/jpgn/Fulltext/2008/11000/Quality of Lif e in Adolescents With Treated.5.aspx

34. Chan FW, Wong FY, So WY, Kung K, Wong CK. How much do elders with chronic conditions know about their medications? BMC Geriatr [Internet]. 2013 [cited 2013 set 25];13:59-65. Available from: http://www.biomedcentral.com/14712318/13/59

35. Souza C, Vedana KGG, Mercedes BPC, Miasso AI. Transtorno bipolar e medicamentos: adesão, conhecimento dos pacientes e monitorização sérica do carbonato de lítio. Rev. Latino-Am. Enfermagem [Internet]. 2013 [cited 2013 set 25];21(2): [8 telas]. Available from:

http://www.scielo.br/pdf/rlae/v21n2/pt_0104-1169-rlae-21-020624.pdf

36. Secretaria de Vigilância em Saúde. Programa Nacional de DST e Aids. Manual de adesão ao tratamento para pessoas vivendo com HIV e AIDS. Brasília (Brasil): Ministério da Saúde; 2008.

37. Van Hecke A, Verhaeghe S, Grypdonck M, Beele H, Defloor T. Processes underlying adherence to leg ulcer treatment: a qualitative field study. Int J Nurs Stud [Internet]. 2011[cited 2013 set 25];48(2):145-55. Available from:

http://www.sciencedirect.com/science/article/pii/S00207489100 $\underline{02129}$

38. Finlayson $K$, Edwards $H$, Courtney $M$. The impact of psychosocial factors on adherence to compression therapy to prevent recurrence of venous leg ulcers. J Clin Nurs [Internet]. 2010 [cited 2013 set 25];19(9-10):1289-97. Available from: http://onlinelibrary.wiley.com/doi/10.1111/j.13652702.2009.03151.x/pdf

39. Nemes MIB, Castanheira REL, Santa-Helena ET, Melhior R, Caraciolo JM, Basso CR, et al. Adesão ao tratamento, acesso e qualidade da assistência em AIDS no Brasil. Rev Assoc Med Bras [Internet]. 2009 [cited 2013 set 25];55(2):207-12. Available from: http://www.scielo.br/pdf/ramb/v55n2/28.pdf 40. Matta SR, Luiza VL, Azeredo TB. Adaptação brasileira de questionário para avaliar adesão terapêutica em hipertensão arterial. Rev Saúde Pública [Internet]. 2013 [cited 2013 set 25];47(2):292-300. Available from: http://www.scielosp.org/pdf/rsp/v47n2/0034-8910-rsp-47-02 0292.pdf

41. Davies MA, Boulle A, Fakir T, Nuttall J, Eley B. Adherence to antiretroviral therapy in young children in Cape Town, South Africa, measured by medication return and caregiver self-report: a prospective cohort study. BMC Pediatr [Internet]. 2008 [cited 2013 set 25$] ; 8(34):[12$ telas]. Available from:

http://www.ncbi.nlm.nih.gov/pmc/articles/PMC2533648/pdf/147 1-2431-8-34.pdf

42. Biadgilign S, Deribew A, Amberbir A, Deribe K. Adherence to highly active antiretroviral therapy and its correlates among HIV infected pediatric patients in Ethiopia. BMC Pediatr [Internet]. 2008 [cited 2013 set 25];8(53):[9 telas]. Available from: http://www.ncbi.nlm.nih.gov/pubmed/19061515

Artigo recebido em 09/01/13.

Aprovado para publicação em 16/09/13.

Artigo publicado em 31/03/2014. 